\title{
Homogenization Technique for the Efficient Modeling of Large Scale Corrugated Core Sandwich Structures
}

\author{
Isabella C. Skrna-Jakl, Dieter H. Pahr, Karl Heinz Karner, and Franz G. Rammerstorfer
}

\begin{abstract}
Numerical calculations of large scale corrugated core sandwich structures are, due to their characteristic cross sections, generally very labor-intensive and costly. With the application of effective orthotropic FE-shell elements, which are based on a homogenization technique, modeling and computation of these structures can be carried out more efficiently. In the present paper an analytical homogenization framework is presented. For the verification of the analytical stiffness predictions a parametric study is performed employing FE-unit cell analyses. Numerical analyses of four-point bending tests employing effective orthotropic shell elements are performed in order to demonstrate the effectiveness of these elements. Finally, the numerical bending test results are verified experimentally.
\end{abstract}

Index Terms-Corrugated core sandwich structures, finite elements, lightweight design, orthotropic material behavior.

\section{INTRODUCTION}

Metro vehicles, passenger coaches or bus body structures are only a small selection from a wide range of applications of corrugated core sandwich (CCS) panels in large scale structures. As CCS panels are effective lightweight integral design components which achieve a significant reduction in mass and assembly costs in comparison to conventional frame structures, their area of application has increased significantly in recent years [1]. In vehicle frame structures CCS panels made of aluminum are employed in the roof, sidewall [2] and floor areas. Typical cross sections are shown in Fig. 1.

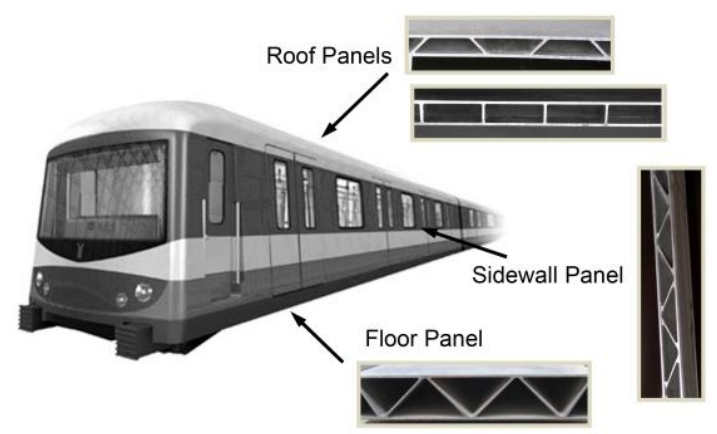

Fig. 1. Typical cross sections of CCS panels in vehicle frame structures.

Manuscript received September 30, 2013; revised January 15, 2014. This work was supported in part by the Hochschuljubiläumsstiftung der Stadt Wien, Projekt -Nr. H-1049/2003

I. C. Skrna-Jakl, D. H. Pahr, and F. G. Rammerstorfer are with the Institute of Lightweight Design and Structural Biomechanics, Vienna University of Technology, 1040 Vienna, Austria (e-mail: skrna@ilsb.tuwien.ac.at, pahr@ilsb.tuwien.ac.at, ra@ilsb.tuwien.ac.at ).

K.-H. Karner is with Siemens AG, Vienna, Austria (e-mail: karl-heinz.karner@siemens.com).
For the numerical analysis of large scale CCS structures isotropic finite shell elements could be applied. However, due to the complex panel cross sections, a detailed modelling of the isotropic face sheets and the interior stiffeners is required, which leads to either huge or too coarse FE-meshes. In order to reduce the modeling input as well as computational requirements, effective, i.e., homogenized, orthotropic shell elements have been developed allowing substantial reduction of degrees of freedom by taking the detailed geometry implicitly into account. These thick shell elements are based on analytical approaches for predicting the complete orthotropic mechanical response of the investigated CCS panels. This paper aims, on the one hand, to present and verify the analytical stiffness approaches and, on the other hand, to show that the application of effective orthotropic shell finite elements significantly increases the efficiency of the analysis of CCS structures.

\section{INVESTIGATED CONFIGURATIONS}

The investigated CCS panels consist of two face layers (fl), which are separated by periodically placed interior stiffeners (st). In the present paper two specific stiffener configurations are studied: in the first type, referred to as "rectangular cross section", all stiffeners are oriented perpendicularly to the face layers, Fig. 2a. The stiffeners of the second type, referred to as "diagonal cross section", are arranged diagonally with a certain inclination to the face sheets, Fig. 2 b.

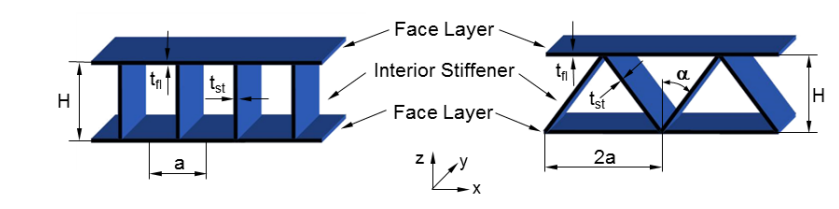

Fig. 2a. Rectangular cross section.

Fig. 2b. Diagonal cross section.

Fig. 2a and Fig. 2b show the cross section parameters for both types. For each investigated configuration both face layers have the same thickness $t_{f l}$ and all interior stiffeners have the thickness $t_{s t}$. The distance between the midplanes of the face layers is $H$. A periodic length is defined with $a$ for the rectangular cross section and $2 a$ for the diagonal cross section. For the face sheets and the interior stiffeners the same linear elastic, isotropic material $(E, G, v)$ is assumed.

\section{EFFECTIVE ORTHOtropic SHELl ELEMENTS}

With the introduction of effective orthotropic shell elements CCS structures can be modeled efficiently: on the one hand, only one shell element over the thickness of the panel is required and, on the other hand, the element size does 
not depend on the position of the interior stiffeners any longer. As a result coarser meshes can be used.

Effective orthotropic shell elements are conventional thick finite shell elements as typically provided by commercial FE-codes. Their orthotropic mechanical behavior is defined via equivalent section properties. With the definition of an appropriate coordinate system, Fig. $2 \mathrm{a}$ and $2 \mathrm{~b}$, the equivalent section properties correspond to the components of the orthotropic effective section stiffness matrix of the material law given in (1).

$$
\left\{\begin{array}{l}
N_{x x} \\
N_{y y} \\
N_{x y} \\
M_{x x} \\
M_{y y} \\
M_{x y} \\
Q_{x z} \\
Q_{y z}
\end{array}\right\}=\left\{\begin{array}{ccc}
A_{11} & A_{12} & 0 \\
A_{21} & A_{22} & 0 \\
0 & 0 & A_{33} \\
& & \\
& & \\
& &
\end{array}\right.
$$$$
\left.\begin{array}{cccccc}
D_{11} & D_{12} & 0 & & \\
D_{21} & D_{22} & 0 & & \\
0 & 0 & D_{33} & & \\
& & & K_{11} & 0 \\
& & & 0 & K_{22}
\end{array}\right\}\left\{\begin{array}{l}
\varepsilon_{x x} \\
\varepsilon_{y y} \\
\gamma_{x y} \\
\kappa_{x x} \\
\kappa_{y y} \\
\kappa_{x y} \\
\gamma_{x z} \\
\gamma_{y z}
\end{array}\right\}
$$

The effective section stiffness matrix is devided into three parts: the effective membrane stiffness matrix $A$, the effective bending stiffness matrix $D$, and the effective transverse shear stiffness matrix $K$. The mathematical vector on the left side consists of the membrane forces $\left(N_{x x}, N_{y y}, N_{x y}\right)$, the bending moments $\left(M_{x x}, M_{y y}, M_{x y}\right)$, and the transverse shear forces $\left(Q_{x z}, Q_{y z}\right)$, all stress resultants are values per unit length. The strain vector on the right side includes the membrane strains in the shell's reference surface $\left(\varepsilon_{x x}, \varepsilon_{y y}, \gamma_{x y}\right)$, the cross section curvatures $\left(\kappa_{x x}, \kappa_{y y}, \kappa_{x y}\right)$ and the transverse shear angles $\left(\gamma_{x z}, \gamma_{y z}\right)$.

The components $A_{i 3}, A_{3 j}, D_{i 3}$ and $D_{3 j}$ for $i \neq 3$ and $j \neq 3$ as well as $K_{12}$ and $K_{21}$ vanish as it is presupposed that the employed coordinate system $(x, y, z)$ coincides with the axes of orthotropy of the investigated CCS panel configurations. The remaining components of the effective section stiffness matrix, which are not specified in (1), are set to zero, because coupling effects are not expected for the investigated cross sections.

In the following subsections a homogenization technique is presented, which uses analytical formulas for the prediction of the components of the effective section stiffness matrices $A, B$ and $K$ of the investigated configurations.

\section{A. Effective Membrane Stiffness Parameters: A11, A12,} A22

For the derivation of the components of the membrane stiffness matrix it is assumed that in $x$-direction the membrane stiffness of the face layers dominates. Thus

Only contributions from the face layers are taken into account when calculating $A_{11}$. This simplifying assumption is true for rectangular cross sections and applicable for diagonal cross sections, too. In (2) to (4) mathematical expressions for $A_{11}, A_{12}, A_{21}$, and $A_{22}$ are given, based on cross section parameters shown in Fig. 2a and Fig. 2b, with $\alpha=0$ for rectangular cross sections.

$$
A_{11}=\frac{2 t_{f l} E}{1-v^{2}}
$$

$$
\begin{aligned}
& A_{12}=A_{21}=\frac{v 2 t_{f l} E}{1-v^{2}} \\
& A_{22}=\frac{2 t_{f l} E}{1-v^{2}}+\frac{H t_{s t} E}{\cos \alpha a}
\end{aligned}
$$

\section{B. Effective Bending Stiffness Parameters: $D_{11}, D_{12}, D_{22}$}

The formulas for the components of the bending stiffness matrix are based on similar assumptions as presented in subchapter A. Therefore, with respect to the local, i.e. member bending stiffness, only these of the face layers are taken into account, while the local bending stiffness of the stiffeners are neglected. Thus, the overall bending stiffness of a CCS panel subjected to $M_{x x}$, is described by the following expressions (5) to (7) for $D_{11}, D_{12}, D_{21}$, and $D_{22}$ :

$$
\begin{gathered}
D_{11}=\frac{E}{1-v^{2}}\left[\frac{t_{f l}{ }^{3}}{6}+\frac{t_{f l} H^{2}}{2}\right] \\
D_{12}=D_{21}=\frac{v E}{1-v^{2}}\left[\frac{t_{f l}{ }^{3}}{6}+\frac{t_{f l} H^{2}}{2}\right] \\
D_{22}=\frac{E}{1-v^{2}}\left[\frac{t_{f l}{ }^{3}}{6}+\frac{t_{f l} H^{2}}{2}\right]+\frac{t_{s t} E H^{3}}{12 a \cos \alpha}
\end{gathered}
$$

\section{Effective Membrane Shear Stiffness Parameter: $A_{33}$}

The static equivalence between the external inplane shear load $N_{x y}$ and the internal shear flows in the face layers and the interior stiffeners of a CCS unit cell is utilized to derive the analytical expression (8) for the membrane shear stiffeness component $A_{33}$.

$$
A_{33}=G\left(2 t_{f l}+t_{s t} \sin \alpha\right)
$$

\section{Effective Twisting Stiffness Parameter: $D_{33}$}

In the investigated CCS panel configurations the applied twisting moment $M_{x y}$ causes only shear flows in the face layers. Due to this fact only the face layers are considered for the derivation of the mathematical expression (9) for twisting stiffness component $D_{33}$.

$$
D_{33}=\frac{G}{6}\left(3 H^{2} t_{f l}+t_{f l}^{3}\right)
$$

\section{E. Effective Transverse Shear Stiffness Parameters: $K_{11}$,} $K_{22}$

As CCS panels, in particular the investigated configurations with rectangular cross sections, show a weak transverse shear behavior in the $x z$-plane, Fig. $2 \mathrm{a}$, transverse shear deformations must be taken into account. For the derivation of the components of the transverse shear stiffness matrix two different approaches for $K_{11}$ and $K_{22}$ are utilized.

For the prediction of $K_{11}$ the deformation behavior of a CCS unit cell of one periodic length is studied analytically. A simplified 2D-beam model of a unit cell cantilever, which is loaded by $Q_{x z}$ at the free edge, is used. After the determination of the distribution of $Q_{x z}$ between the top and the bottom face layers of the unit cell, the force-method is applied to solve the statically indeterminate $2 \mathrm{D}$-system. In a 
second step analytical expressions for the displacements in $z$-direction at the load introduction points are derived by applying Castigliano's second theorem. The determined displacements $(w)$ in $z$-direction include the transverse shear $\left(w_{s}\right)$ and bending deformations $\left(w_{b}\right)$ at the free edge of the unit cell. With the use of formulas of the beam theory for $w_{s}$ and $w_{b}$ of a cantilever and considering the assumptions for the bending stiffness, presented in subchapter B, a mathematical expression for $K_{11}$ can be derived. In this step also all membrane and bending displacements are multiplied by $\left(1-v^{2}\right)$ in order to make $K_{11}$, which was derived by employing 2D-beam models, applicable for shells. As the presented way of obtaining $K_{11}$ of the diagonal cross section leads to very long expressions, only the formula $K_{11, r}$ of the rectangular cross section is given (10).

$$
K_{11, r}=\left(\begin{array}{l}
\frac{a^{2}\left(1-v^{2}\right)}{E t_{f l} H^{2}}+\frac{6(1+v)}{5 E t_{f l}}+\frac{12 a(1+v)}{5 E t_{s t} H}+ \\
+\frac{a^{2}\left(1-v^{2}\right)}{2 E t_{f l}^{3}}+\frac{a H\left(1-v^{2}\right)}{E t_{s t}^{3}}-\frac{2 a^{2}\left(1-v^{2}\right)}{E t_{f l}\left(t_{f l}^{2}+3 H^{2}\right)}
\end{array}\right)^{-1}
$$

The prediction of $K_{22}$ is also based on formulas which were developed for cantilevers [3] and which are applied to unit cell models loaded by $Q_{y z}$ at the free edge. The mathematical expressions of the effective transverse stiffness parameters for the rectangular cross section $K_{22, r}$ and the diagonal cross section $K_{22, d}$ are given in (11) and (12), respectively. $J_{x}$ is the second area moment of inertia of one periodic length of the cross section.

$$
\begin{aligned}
& K_{22, r}=\frac{E J_{x}^{2}}{2 a(1+v)} \\
& \left(\int_{0}^{H} \frac{1}{t_{s t}}\left(\frac{H t_{f l} a}{2}+\frac{H t_{s t} s}{2}-\frac{t_{s t} s^{2}}{2}\right)^{2} d s+\frac{H^{2} t_{f l} a^{3}}{24}\right)^{-1} \\
& K_{22, d}=\frac{E J_{x}^{2}}{2 \cdot a \cdot(1+v)} \\
& \left(\int_{0}^{H / \cos \alpha} \frac{4}{t_{s t}}\left(\frac{H t_{f l} a}{2}+\frac{H t_{s t} s}{2}-\frac{t_{s t} s^{2} \cos \alpha}{2}\right)^{2} d s+\frac{2 H^{2} t_{f l} a^{3}}{3}\right)^{-1}
\end{aligned}
$$

\section{VERIFICATION AND PARAMETRIC STUDY}

For the verification of the analytically predicted effective stiffness values finite element unit cell analyses of the investigated CCS panels were performed [4].

The effective membrane and bending stiffness parameters are obtained numerically employing the in-house homogenization software MEDTOOL [5]. In order to compare the analytical stiffness values with the numerical results a parametric study is carried out. Significant cross section parameters are selected and varied within an appropriate range.

The parameters of the diagonal cross section are the inclination $\alpha$ of the interior stiffeners in a range of $10^{\circ} \leq \alpha \leq$ $60^{\circ}$, the distance between the face layers $\mathrm{H}$ in a range of
$20 \mathrm{~mm} \leq H \leq 80 \mathrm{~mm}$ and the ratio between the thickness of the face layers and the interior stiffeners $t_{f} / t_{s t}$, which is set to the values of 1 and 2, respectively. The stiffener thickness to the distance of the face layers ratio is set to $t_{s t} / H=0.05$.
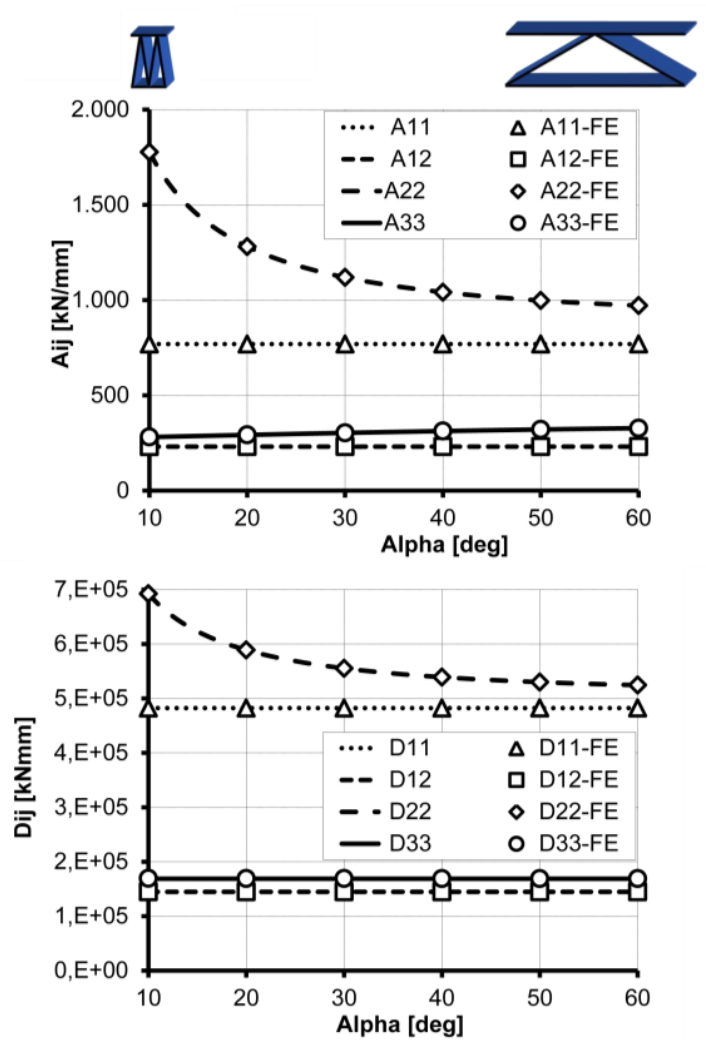

Fig. 3. Comparison of the analytical $\left(A_{i j}, D_{i j}\right)$ and the numerical $\left(A_{i j}\right.$-FE, $D_{i j}$-FE) stiffness components of the diagonal cross section $\left(10^{\circ} \leq \alpha \leq 60^{\circ}\right.$, $\left.H=50 \mathrm{~mm}, t_{f l} / t_{s l}=2\right)$.

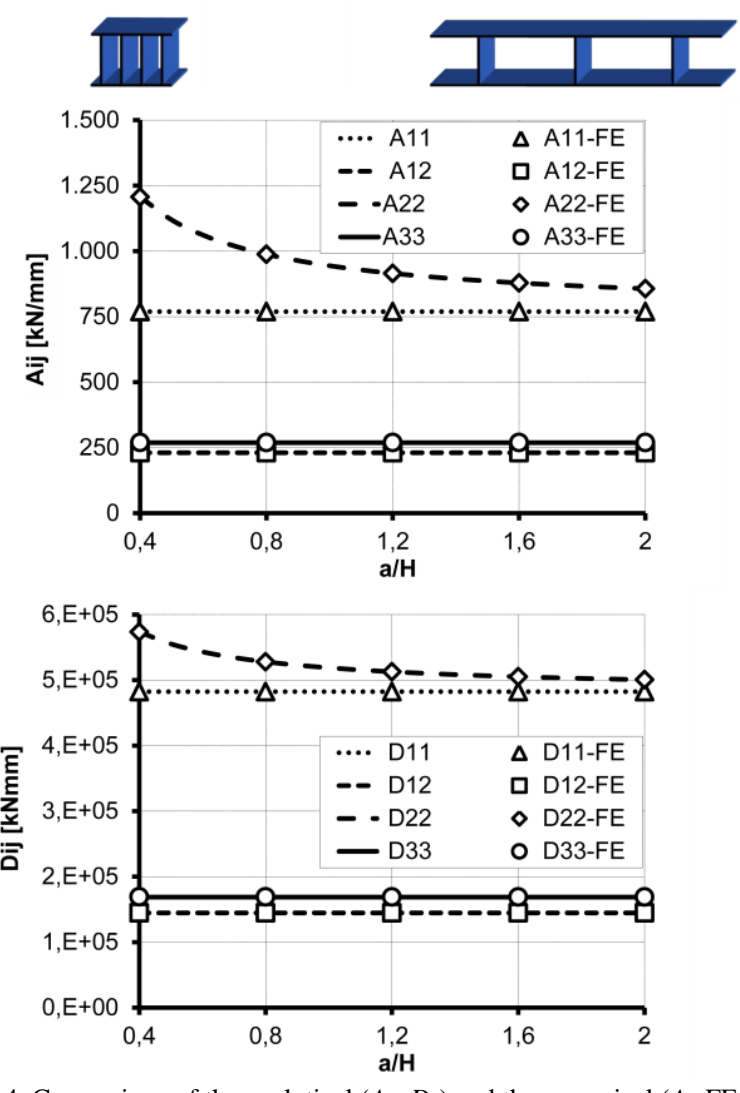

Fig. 4. Comparison of the analytical $\left(A_{i j}, D_{i j}\right)$ and the numerical $\left(A_{i j}-\mathrm{FE}\right.$, $D_{i j}$-FE) stiffness components of the rectangular cross section $(0.4 \leq a / H \leq 2$, $\left.H=50, t_{f} / t_{s t}=2\right)$. 
For the rectangular cross section the following parameters are investigated: the ratio of the periodic length $a$ to the distance between the face layers $H$ in a range of $0.4 \leq a / H \leq 2$ and $H$ in a range of $20 \mathrm{~mm} \leq H \leq 80 \mathrm{~mm}$. The values of $t_{f l} / t_{s t}$ and $t_{s} / H$ correspond to the values of the diagonal cross section.

The results of the parametric study show a good agreement between the analytically and numerically predicted stiffness values in the considered parameter range, Fig. 3 and Fig 4. A maximum variation of less than $1 \%$ is obtained. Due to this outcome the graphs of only two variations of parameters are shown. A detailed description of the results of the complete parametric study is given in [5].

Equivalent cross section parameters are used to present the comparison of the numerical and analytical results of the components of the transverse stiffness matrix, $K_{11}$ and $K_{22}$, Fig.5 and Fig.6. In this case, too, good agreement can be observed. However, especially at the limits of the employed cross section ranges, deviations between the numerical and analytical values start to grow. A maximum deviation of about $7 \%$ for $K_{22}$ at an inclination angle of $60^{\circ}$ of the diagonal cross section is observed. It is also worth mentioning that, in contrast to the values of the membrane and bending stiffness components, $K_{11}$ and $K_{22}$ of the rectangular cross section and $K_{22}$ of the diagonal cross section decrease significantly with increasing values of $\alpha$ and $a / H$, respectively. The results for $K_{11}$ of the diagonal cross section show that the transverse shear stiffness values in the $x z$ plane can be maximized depending on the inclination of the interior stiffeners. Especially for the rectangular cross section the small values of $K_{11}$ point out their weak transverse shear stiffness behavior in the $x z$ plane.
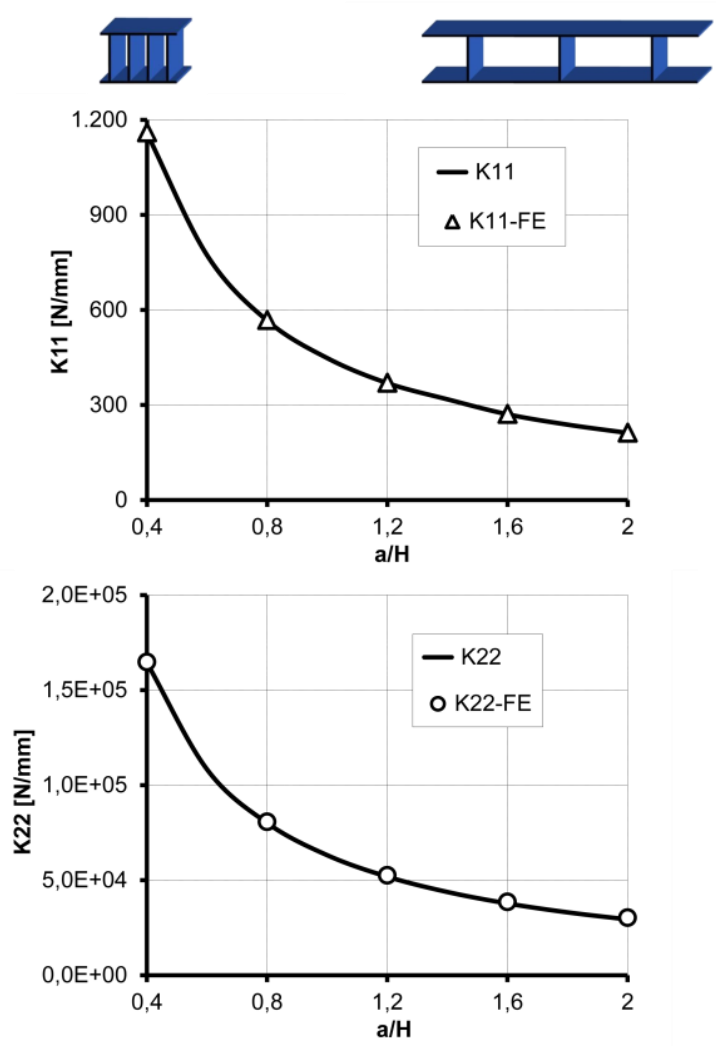

Fig. 5. Comparison of the analytical $\left(K_{11}, K_{22}\right)$ and the numerical $\left(K_{11}-\mathrm{FE}\right.$, $\left.K_{22}-\mathrm{FE}\right)$ transverse shear stiffness components of the diagonal cross section, $\left(10^{\circ} \leq \alpha \leq 60^{\circ}, H=50 \mathrm{~mm}, t_{f l} / t_{s t}=2\right)$.
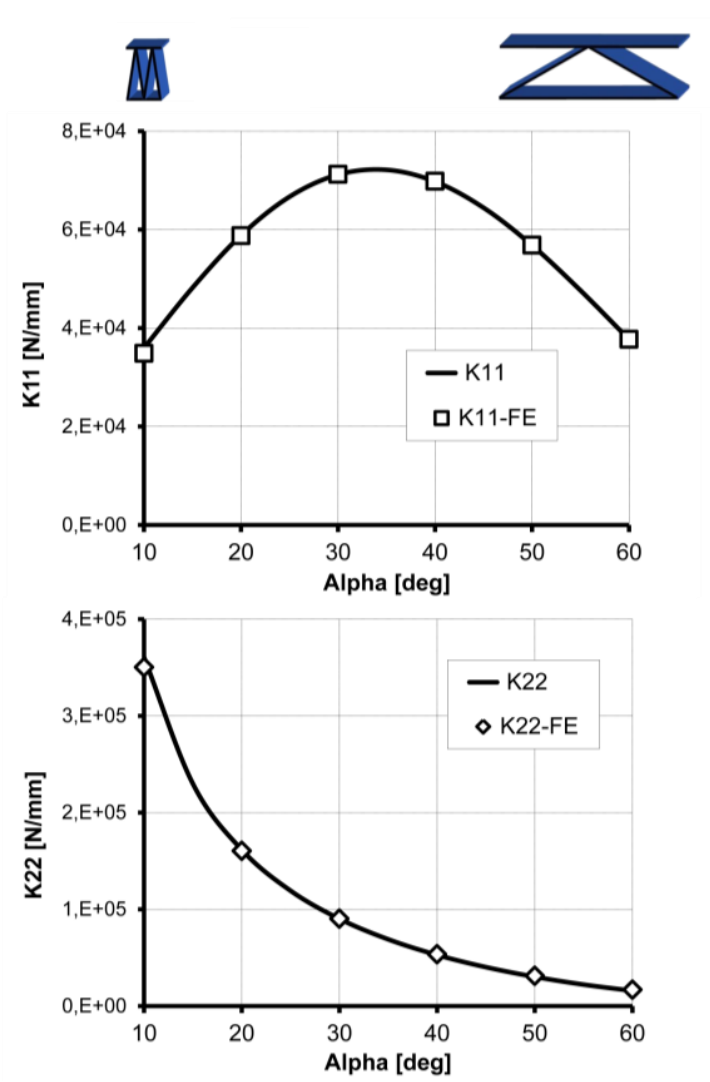

Fig. 6. Comparison of the analytical $\left(K_{11}, K_{22}\right)$ and the numerical $\left(K_{11}-\mathrm{FE}\right.$, $K_{22}$-FE) transverse shear stiffness components of the rectangular cross section, $\left(0.4 \leq a / H \leq 2, H=50, t_{f l} / t_{s l}=2\right)$.

\section{APPLICATION OF EFFECTIVE ORTHOTROPIC SHELL ELEMENTS}

In order to study the efficiency of the developed effective orthotropic shell elements, finite element analyses of four-point bending tests were performed. The specimens were modeled, on the one hand, in very detail with isotropic shell elements and, on the other hand, with effective orthotropic shells. The results of six finite element analyses of models with rectangular cross sections are discussed in the following. All investigated samples have the same dimensions and are transversally stiffened, Fig. 7.

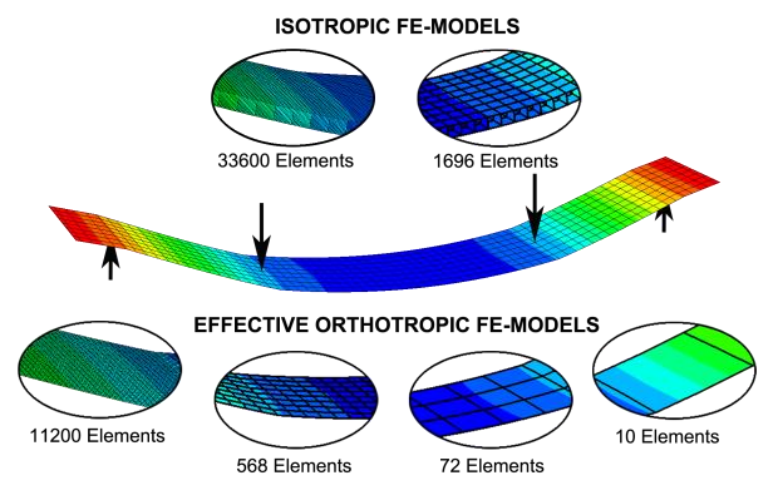

Fig. 7. Four-point bending tests of transversally stiffened specimens used in the FE analyses.

For all FE-analyses the FE-Code ABAQUS [6] was used. First a fine mesh and a coarse mesh, employing 8-noded isotropic shell elements were analyzed. In the fine mesh (33600 elements) four elements are applied along the stiffener height and in the face layers between the stiffeners. 
The coarse mesh (1696 elements) uses the minimum number of isotropic shell elements with one element per stiffener height and one element in the face layers between the stiffeners, Fig. 7. For the isotropic shell models the number of elements depends strongly on the position of the interior stiffeners. By employing 8-noded effective orthotropic thick FE-shell elements coarser meshes can be utilized. In the present study, on the one hand, two effective orthotropic FE-models (11200 and 568 elements), which use a mesh size corresponding to the isotropic models, are studied. On the other hand, two effective orthotropic FE-models (72 and 10 elements) are analyzed which employ far coarser meshes. A comparison of the results of the investigated specimens regarding the number of variables, CPU time and maximum displacements are given in Table I.

TABLE I: COMPUTATIONAL DATA

\begin{tabular}{llrrr}
\hline \hline Elements & FE-Type & Variables & CPU & Max. Displ. \\
\hline 33600 & S8R5 isotropic & 783432 & $82,0 \mathrm{~s}$ & $100 \%$ \\
1696 & S8R5 isotropic & 36312 & $3,04 \mathrm{~s}$ & $99,6 \%$ \\
11200 & S8R eff. orthotropic & 34241 & $11,2 \mathrm{~s}$ & $100,4 \%$ \\
568 & S8R eff. orthotropic & 11178 & $0,43 \mathrm{~s}$ & $100,4 \%$ \\
72 & S8R eff. orthotropic & 1626 & $0,12 \mathrm{~s}$ & $100,4 \%$ \\
10 & S8R eff. orthotropic & 318 & $0,11 \mathrm{~s}$ & $100,4 \%$ \\
\hline \hline
\end{tabular}

The computational data in Table I show that with the application of effective orthotropic shell elements the numerical effort can be reduced significantly, with very little influence on the displacements. This demonstrates the high efficiency of the developed effective orthotropic shell elements for the application in large scale CCS structures.

\section{EXPERIMENTAL VERIFICATION}

For the verification of the numerically predicted displacement behavior of the four-point bending tests, corresponding experimental tests were performed. The four-point bending test setup is shown in Fig. 8. Specimens made of Polycarbonate with rectangular cross sections, see Fig. 9, and stiffeners in transversal and longitudinal direction were investigated, respectively.

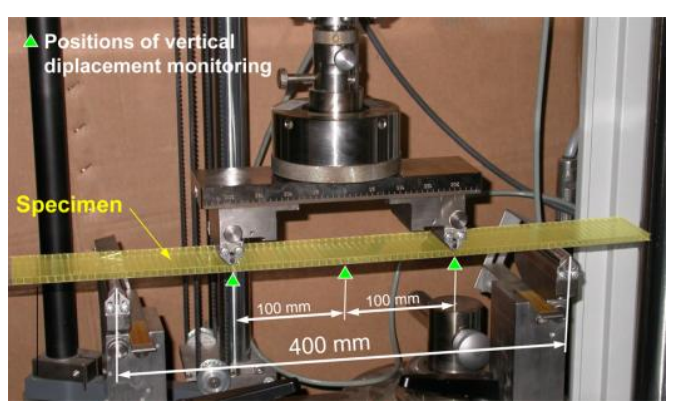

Fig. 8. Four-point-bending test setup.

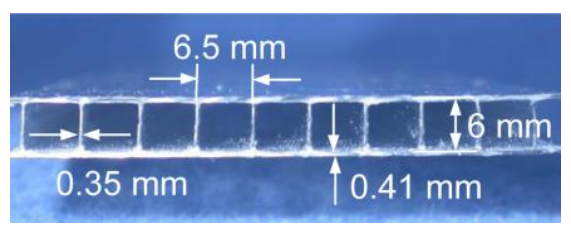

Fig. 9. Sample cross section.

In Fig. 10 and Fig. 11 the experimentally measured and numerically predicted test results, employing an isotropic shell FE-model and an effective orthotropic shell FE-model, are compared. The displayed deformed shapes of the transversally stiffened specimens (Fig. 10) show that the transverse shear deformations, observed in the areas between the supports and the load applications, of the specimen have a major influence on its overall displacements. Furtheron, the comparison of the measured load displacement curves in the center of the specimens (marked with a red arrow) and the corresponding results of the FE-Analyses show a good agreement.
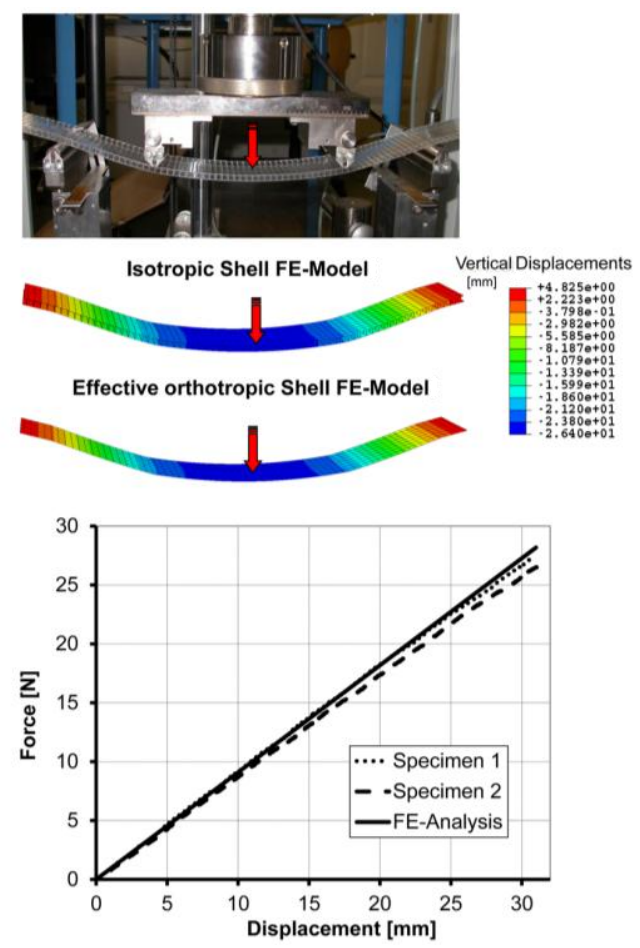

Fig. 10. Deformed shapes and load-displacement curves evaluated in the center (red arrow) of the transversally stiffened specimen.
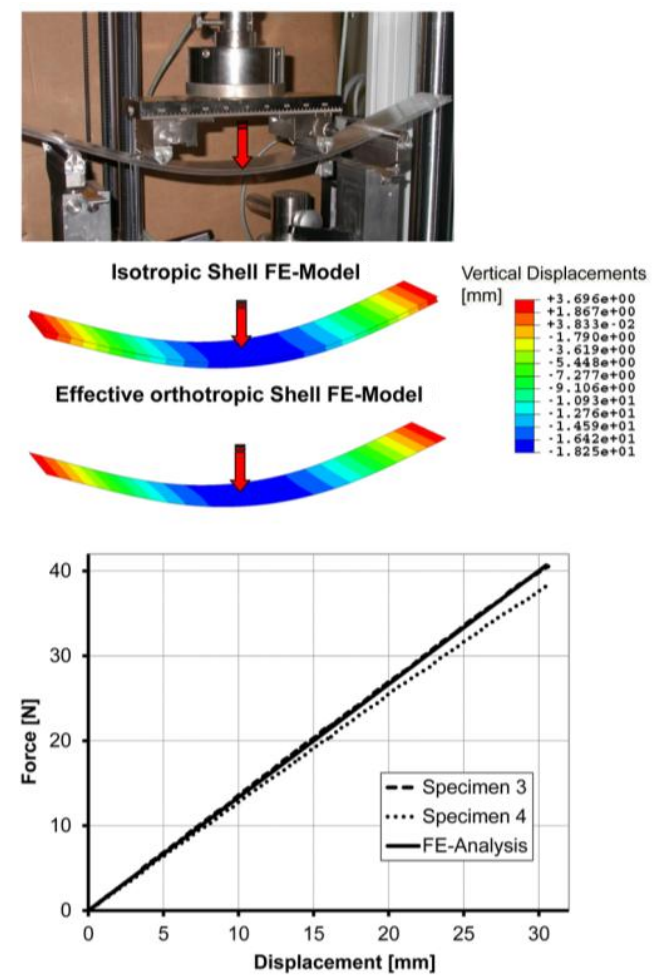

Fig. 11. Deformed shapes and load-displacement curves evaluated in the center (red arrow) of the longitudinally stiffened specimen. 
The numerical and experimental results of the four-point bending tests of the longitudinally stiffened specimens are shown in Fig. 11. In this case it can be observed that the displayed deformations are dominated by bending. The diagram in which the measured and predicted vertical displacements at the center point of the samples (indicated by a red arrow) are displayed, also shows the good correlation between the numerical and experimental results.

In both test configurations a maximum deviation of less than $5 \%$ between the numerical and experimental values of the central displacements was detected.

\section{CONCLUSION}

A homogenization technique, which is based on analytical approaches, is presented. The analytically predicted stiffness values are verified for a range of geometrical parameters of two typical CCS cross sections by employing finite element unit cell analyses. The results show a deviation of less than $1 \%$ for the membrane and bending stiffness components. For the transverse shear stiffness parameters also a good correlation between the numerical results and the analytical values is obtained. The results of the homogenization technique are used to define effective orthotropic shell elements. In order to prove their efficiency, finite element analyses of four point bending tests of transversally stiffened CCS panels are performed. A comparison of the results and the computational data of the coarse isotropic shell model (1696 elements) and the corresponding effective orthotropic shell model (348 elements) show that, with the application of effective orthotropic shells the number of variables can be reduced to $30 \%$ and the CPU time to $14 \%$ of the isotropic shell model, when the same mesh-size is used. If a coarser mesh is applied in the effective orthotropic shell models the number of variables can even be reduced to $0.6 \%$ and the CPU time to $3.6 \%$. The comparison of the maximum displacements of all models shows a deviation of less than $1 \%$. These results show clearly the high efficiency of the developed effective orthotropic shell elements for modeling corrugated core sandwich panels.

An experimental study of transversally and longitudinally stiffened specimens, employing four-point bending tests, also verified the applicability of the developed effective orthotropic shell elements.

As far as local stresses are concerned, the stress resultants calculated by models using effective orthotropic shell elements can be used in a localization procedure, i.e. by inverting the homogenization.

\section{ACKNOWLEDGMENTS}

The support of the Institute of Materials Science and Technology and Gutta Werke GmbH, Schutterwald, Germany is gratefully acknowledged.

\section{REFERENCES}

[1] T. Evertz et al., "Die Leichtbauwerkstoffe für den Fahrzeugbau," in Leichtbau in der Fahrzeugtechnik, H. E. Friedrich, Ed., Wiesbaden Springer Vieweg, 2013, ch. 6.2, pp. 280-285.

[2] L. Zheng, D. Petry, T. Wierzbicki, and H. Rapp, "Fracture prediction in 4-point bending of an extruded aluminum panel," Thin walled Structures, vol. 43, pp. 565-590, 2005.

[3] H. Mang and G. Hofstetter, Festigkeitslehre, Wien: Springer-Verlag, 2008, ch. 6.7.6.

[4] K. H. Karner, "Homogenisierung und Lokalisierung bei der Modellierung von periodisch strukturierten, schalenartigen Strangpressprofilen,” M.S. thesis, Dept. Mech. Eng., Vienna Univ. of Technology, Vienna, Austria, 2005.

[5] D. H. Pahr and F. G. Rammerstorfer, "Buckling of honeycomb sandwiches: periodic finite element," CMES Computer Modeling in Engineering \& Science, vol. 12, pp. 229-242, July 2006.

[6] ABAQUS v 6.13, (C) Dassault Systems Simulia Corp., Providence, RI, 2013.

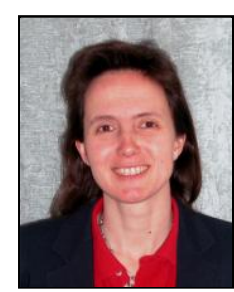

Isabella C. Skrna-Jakl studied mechanical engineering at the Vienna University of Technology (TU Vienna), Vienna, Austria, where she graduated in 1989. At the Institute of Lightweight Design and Aerospace Engineering of the TU Vienna she finished her PhD in December 1994.

She worked as a practical trainee at the Technical Department of Austrian Airlines and at Fischer Advanced Composite Components. After her graduation she took the position as development engineer and stress analyst at the Austrian Aerospace Company. Since 1991 she has been employed as University Assistant and since 2003 as Assistant Professor at the Institute of Lightweight Design and Structural Biomechanics at the TU-Vienna, Vienna, Austria. Her current research interests are the numerical and analytical simulation of the mechanical behavior of lightweight structures focusing on sandwich structures as well as Composite materials and structures.

Dr. Skrna-Jakl is a recipient of the 1994 Amelia Earhart Fellowship Award from the Zonta International Foundation. 